\title{
Designing metamaterials with quantum annealing and factorization machines
}

\author{
Koki Kitai $\odot,{ }^{1, *}$ Jiang Guo, ${ }^{2, *}$ Shenghong Ju $\odot,{ }^{2,3}$ Shu Tanaka $\odot,{ }^{4,5}$ Koji Tsuda $\odot,{ }^{1,3,6, \dagger}$ \\ Junichiro Shiomi, $2,3,6, \ddagger$ and Ryo Tamura ${ }^{1,3,6,7, \S}$ \\ ${ }^{1}$ Graduate School of Frontier Sciences, The University of Tokyo, Chiba 277-8568, Japan \\ ${ }^{2}$ Department of Mechanical Engineering, The University of Tokyo, Tokyo 113-8654, Japan \\ ${ }^{3}$ Research and Services Division of Materials Data and Integrated System, National Institute for Materials Science, Ibaraki 305-0047, Japan \\ ${ }^{4}$ Green Computing Systems Research Organization, Waseda University, Tokyo 162-0042, Japan \\ ${ }^{5}$ JST, PRESTO, Saitama 332-0012, Japan \\ ${ }^{6}$ RIKEN Center for Advanced Intelligence Project, Tokyo 103-0027, Japan \\ ${ }^{7}$ International Center for Materials Nanoarchitectonics, National Institute for Materials Science, Ibaraki 305-0044, Japan
}

(Received 12 August 2019; accepted 19 February 2020; published 16 March 2020)

\begin{abstract}
Automated materials design with machine learning is increasingly common in recent years. Theoretically, it is characterized as black-box optimization in the space of candidate materials. Since the difficulty of this problem grows exponentially in the number of variables, designing complex materials is often beyond the ability of classical algorithms. We show how quantum annealing can be incorporated into automated materials discovery and conduct a proof-of-principle study on designing complex thermofunctional metamaterials. Our algorithm consists of three parts: regression for a target property by factorization machine, selection of candidate metamaterial based on the regression results, and simulation of the metamaterial property. To accelerate the selection part, we rely on the D-Wave 2000Q quantum annealer. Our method is used to design complex structures of wavelength selective radiators showing much better concordance with the thermal atmospheric transparency window in comparison to existing human-designed alternatives.
\end{abstract}

DOI: 10.1103/PhysRevResearch.2.013319

\section{INTRODUCTION}

Further evolution in materials that controls energy carriers, such as photons, electrons, and phonons, is a condition to realize sustainable industry and society. The key is to manipulate transport properties at the scale of characteristic length of energy carriers. For the last decades, metamaterial industries have realized top-down fabrications, bottom-up syntheses, and atomistic and spectroscopic characterizations. These advances have given us access to a nearly unlimited exploration of structures with enhanced energy transport characteristics. This situation has resulted in many breakthroughs in photovoltaics [1], thermal radiators [2,3], batteries [4], thermoelectrics [5], and others. Here, metamaterials are a representative case where the artificial structure produced inside a material gives rise to extraordinary properties.

Currently, the major obstacle is that there are too many candidate structures to explore. For example, exploring single crystals is exhausting when considering compounds, but the

\footnotetext{
*These authors equally contributed to this paper.

$\dagger$ tsuda@k.u-tokyo.ac.jp

‡shiomi@photon.t.u-tokyo.ac.jp

stamura.ryo@nims.go.jp
}

Published by the American Physical Society under the terms of the Creative Commons Attribution 4.0 International license. Further distribution of this work must maintain attribution to the author(s) and the published article's title, journal citation, and DOI. number of candidates becomes truly massive when extending to include composites in a broad sense with nanoscale inhomogeneity in the composition or simply "nanostructures." However, some materials with such a compositional inhomogeneity exhibit superior properties compared to their counterparts with ordered structures, particularly in the case where the structures are smaller than the coherence length of energy carriers as shown for photons [6], phonons [7], electrons [8], and magnons [9]. Therefore, for further evolution of materials in energy technology, it is imperative to overcome the challenge of massive candidates. One solution is automated materials discovery.

Automated materials discovery based on black-box optimization is an iterative process to select one candidate from a massive number of candidates (i.e., design space) for experimental investigations [10-12]. From existing materials properties data, machine learning predicts the properties of unobserved candidates and defines an acquisition function in design space. The global optimization problem in design space is solved with respect to this acquisition function. The best candidate is selected as the next candidate material for experiments. Using the observed properties of the selected candidate, the machine learning model is updated and defines a different acquisition function for the next iteration. Repeating this procedure with the aid of the machine learning should drastically reduce the number of experimental investigations to design materials with the desired properties.

There are two barriers that hinder applying automated materials discovery to complex materials design: statistical and computational. A statistical barrier refers to difficulty 


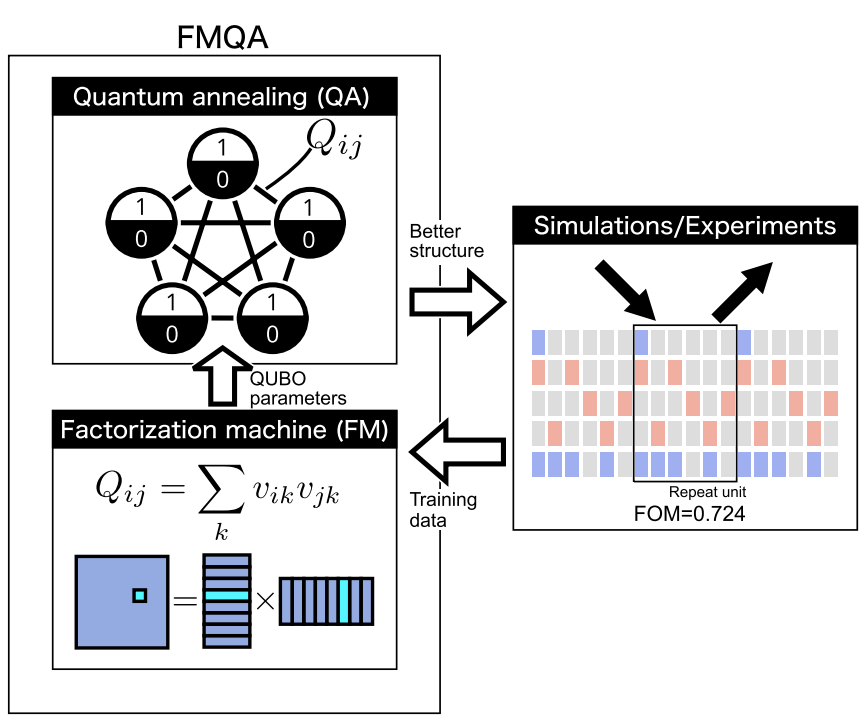

FIG. 1. Procedure of our automated materials discovery using a factorization machine (FM) for regression and quantum annealing (QA) for selection. This simulation can use various simulation methods or experiments, depending on the target properties.

predicting the properties of materials with a limited number of training data by machine learning. A computational barrier is related to the well-known hardness of the global optimization defined by the acquisition function. The statistical barrier is reduced by using a fast simulator, which calculates the properties of the target materials, instead of an experiment. As several recent studies show, a simulation is not as overwhelming as the computational barrier $[10,12]$. To circumvent the difficulty with global optimization, exhaustive searches have been replaced by methods such as local searches, tree searches [13], and genetic algorithms [14]. Because these alternative methods have a lower optimization quality, other options continue to be investigated.

To overcome this computational barrier, we propose a new algorithm that employs a D-Wave quantum annealer [15]. This computer can obtain highly accurate solutions of a particular type of combinatorial optimization called quadratic constrained binary optimization (QUBO) by the quantum annealing (QA) process [16-18]. Many studies have discussed the computational performance of this computer from theoretical and experimental viewpoints [19-25]. To date, diverse applications have been reported in biological science [26,27], machine learning [28-30], and Internet-ofThing (IoT) [31-33].

Figure 1 depicts the schematic procedure of our algorithm. It can solve a black-box optimization problem over binary variables representing the structure of materials. First, a factorization machine (FM) [34] is trained with the available data to model the material property of interest. In this study, the target property is the figure-of-merit (FOM) for radiative sky cooling, which is evaluated by rigorous coupled wave analysis (RCWA). Selection of a new candidate with respect to an acquisition function based on a trained FM comes down the QUBO solved by a quantum annealer. The properties of the new candidate are obtained by RCWA. Then the pair of new candidate and its property is added to training data, and the
FM is retrained. We call our algorithm FMQA (factorization machine with quantum annealing) [35].

As a proof-of-principle, we apply our method to design a metamaterial with tailored thermal radiation spectrum. Tailoring the thermal radiation is fundamentally important because every material emits and absorbs thermal radiation. From an engineering viewpoint, diverse applications require wavelength-selective thermal radiation (photons). For instance, engineered thermal-emission leads to high-efficiency thermophotovoltaics [36,37], incandescent light sources [38], biosensing [39,40], microbolometers [41,42], imaging [43], and drying furnace [44].

Another application that has recently attracted much attention, in response to concerns of global warming and energy crises, is radiative sky cooling that utilizes the untapped $3 \mathrm{~K}$ cold space as a heat sink. Previous designs on radiative cooling [2,45-55] have focuses on simultaneously blocking solar energy $(0.4-4 \mu \mathrm{m})$ while maximizing thermal radiation loss $(>4 \mu \mathrm{m})$ to the surroundings. These designs have been experimentally demonstrated to be successful in dry and clear weather. However, this design strategy is not as effective in hot and humid areas because the atmospheric window $(8-13 \mu \mathrm{m})$, which allows thermal radiation to directly transmit to the outer space, becomes less transparent and a large part of the downward radiation that is beyond the window is absorbed by the radiator. Therefore, a radiator that only emits thermal radiation to outer space through the transparency window is desirable. Such a radiator can help maximize the outgoing radiative cooling power while minimizing the ambient radiative energy absorption. In this study, our proposed optimization method is used to design such a radiator with a wavelength selectivity higher than previously designed ones.

\section{TARGET METAMATERIALS AND METHODS USED IN FMQA}

\section{A. Target metamaterials}

A metamaterial, which only emits or absorbs the thermal radiation within the transparency window of the atmosphere $(8-13 \mu \mathrm{m})$, is preferable for radiative sky cooling [49,53]. Various material structures have been proposed to match the spectrum of the atmospheric window, including planar multilayer structures [2,48], patterned meta-surface structures $[47,50,51,54]$, and polymers doped with nanoparticles $[45,52,55]$. Most of these structures do not have a high emittance over the whole span of the atmospheric window. Additionally, their cooling capability is insufficient. On the other hand, our design employs $\mathrm{SiO}_{2}$ and $\mathrm{SiC}$ to achieve this stringent spectral selectivity. The dielectric functions of $\mathrm{SiO}_{2}$ and $\mathrm{SiC}$ indicate that they have phonon-polariton resonances positioned at 9.7 and $12.5 \mu \mathrm{m}$, respectively. Moreover, they both have very small extinction coefficients in the solar energy wavelength band, implying that the absorption of solar energy should be suppressed.

Inspired by previous research [46], the target metamaterial structure is comprised of $\mathrm{SiO}_{2}$ and $\mathrm{SiC}$ wires placed in poly(methyl methacrylate) (PMMA), which has a negligible absorption in the visible to far-infrared range. Each wire is arranged along the $y$ axis, and light is incident from the top 


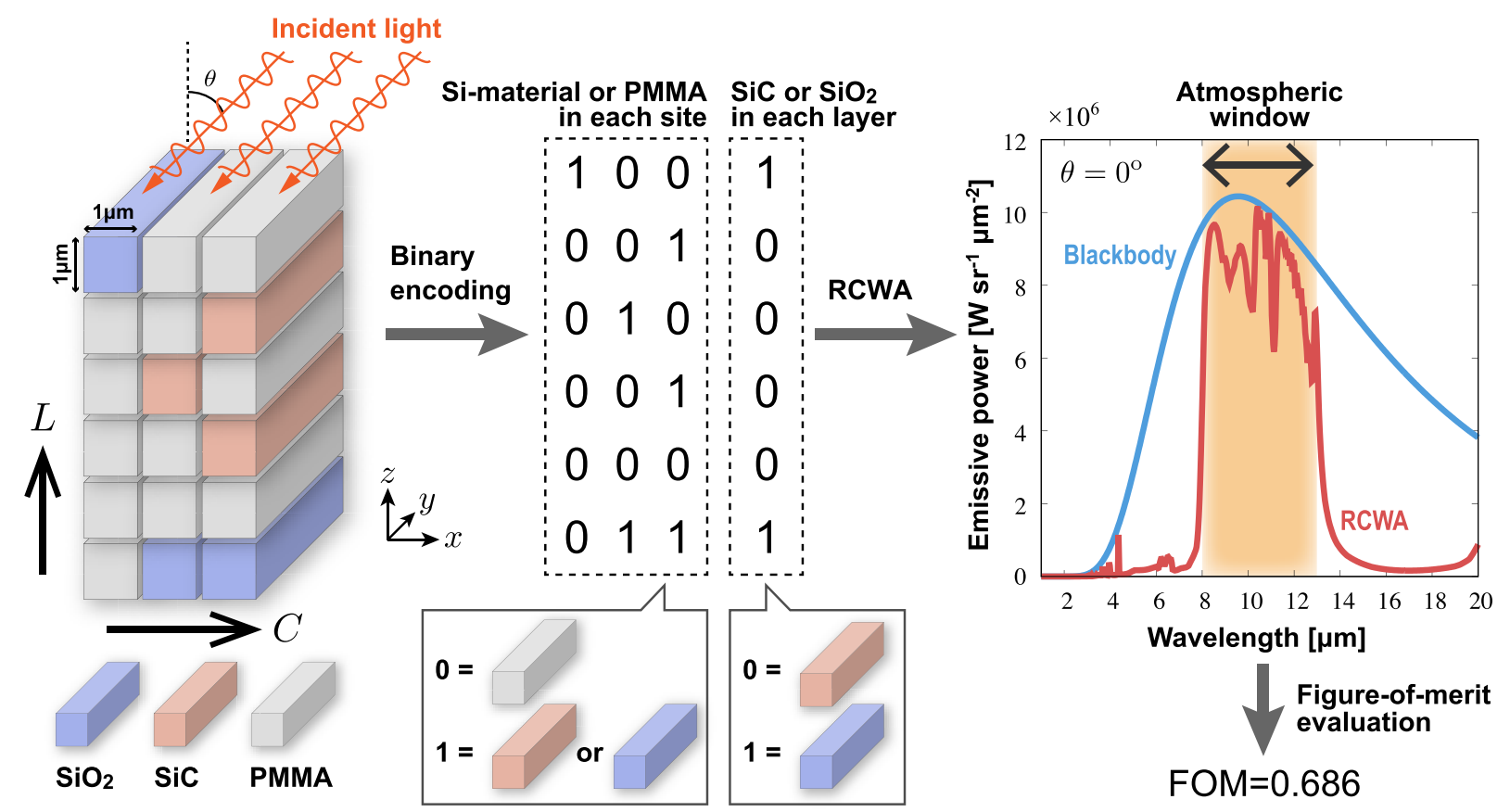

FIG. 2. Example of the target metamaterial structure for $L=6$ and $C=3$, the binary variables expressing it, and the emissive powers of target metamaterial (red curve) and blackbody (blue curve) calculated by RCWA.

layer (Fig. 2). The periodic boundary condition is applied in the $x$ directions (i.e., the structure repeats along the $x$ axis). Because the RCWA calculation solves a two-dimensional $(x-z)$ problem, the wire is assumed to be infinitely long without any variations in the cross section in the $y$ direction. For structural optimization, the $x-z$ plane is uniformly discretized into square units with $1 \mu \mathrm{m}$ side lengths, which are either $\mathrm{SiO}_{2}, \mathrm{SiC}$, or PMMA. The numbers of meshes along the $z$ and $x$ directions are defined as $L$ and $C$, respectively.

One constraint adopted in this study is that a layer can contain only $\mathrm{SiO}_{2}$ or $\mathrm{SiC}$ exclusively. The constraint and the unit size, which correspond to the minimum wire size, are determined considering future fabrication via photolithography. Pretrial simulations confirm that a unit size of $1 \mu \mathrm{m}$ provides sufficient resolution for the optimization problem. For these structures, the emissivity properties are calculated based on RCWA, and the FOM for radiative cooling is evaluated. In our optimization, the target is the emittance property under $p$-polarized incidence (TM wave) for a polar angle $\theta=0^{\circ}$.

To utilize a quantum annealer for automated materials discovery, the metamaterial structure should be encoded into binary variables. Initially, the configuration of the wired materials $\left(\mathrm{SiO}_{2}\right.$ or $\left.\mathrm{SiC}\right)$ and PMMA is determined by $L \times C$ bits (Fig. 2). Then additional bits, which express the type of wired materials in each layer, are prepared. Consequently, the structure of a metamaterial is well-defined using $L \times(C+1)$ bits. Note that our algorithm can perform a structural optimization where $L$ and $C$ are fixed.

\section{B. Simulation by rigorous coupled wave analysis}

To calculate the thermal emissivity properties of the target metamaterials (Fig. 2), RCWA is employed. RCWA is a semi-analytical method to solve Maxwell's equation and provides a high numerical accuracy (see Supplemental note A in Ref. [56]). The spatial distribution of the dielectric constant and the involved electromagnetic field are decomposed in the $x$ and $z$ directions. By imposing a periodic boundary condition in the $x$ direction and a continuous boundary condition in the $z$ direction, the governing Maxwell's equation can be solved quickly and accurately.

Although PMMA is not universal in identical materials due to the influence of the fabrication process, our calculations assume that the PMMA is pure and the refractive index is fixed as 1.48 for simplicity [57]. The dielectric functions of $\mathrm{SiO}_{2}$ and $\mathrm{SiC}$ are obtained from the tabulated data from Palik [58] with interpolation. Comparing the experimental results validates [59] that the RCWA method is a credible approach to design metamaterials for thermal radiators. Figure 2 shows a calculated example of the emissive power.

For a good thermal radiator for radiative cooling, the emittance spectra should fall within the wavelength region between 8 and $13 \mu \mathrm{m}$. To evaluate the likelihood that the designed metamaterial is the ideal case, the FOM is defined as [3]

$$
\mathrm{FOM}=\frac{\int_{\lambda_{\mathrm{i}}}^{\lambda_{\mathrm{f}}} \epsilon_{\lambda} E_{b \lambda} d \lambda}{\int_{\lambda_{\mathrm{i}}}^{\lambda_{\mathrm{f}}} E_{b \lambda} d \lambda}-\frac{\int_{\lambda_{\min }}^{\lambda_{\mathrm{i}}} \epsilon_{\lambda} E_{b \lambda} d \lambda}{\int_{\lambda_{\min }}^{\lambda_{\mathrm{i}}} E_{b \lambda} d \lambda}-\frac{\int_{\lambda_{\mathrm{f}}}^{\lambda_{\max }} \epsilon_{\lambda} E_{b \lambda} d \lambda}{\int_{\lambda_{\mathrm{f}}}^{\lambda_{\max }} E_{b \lambda} d \lambda},
$$

where $\lambda_{\mathrm{i}}=8 \mu \mathrm{m}, \lambda_{\mathrm{f}}=13 \mu \mathrm{m}, \lambda_{\min }=1 \mu \mathrm{m}$, and $\lambda_{\max }=20 \mu \mathrm{m}$. Here, $\epsilon_{\lambda}$ and $E_{b \lambda}$ are the spectral emittance and the spectral blackbody emissive power calculated by RCWA, respectively.

In Eq. (1), $\int_{\lambda_{\mathrm{f}}}^{\lambda_{\mathrm{f}}} \epsilon_{\lambda} E_{b \lambda} d \lambda$ denotes the actual energy heat flux radiated within the wavelength range $8-13 \mu \mathrm{m}$. Furthermore, $\int_{\lambda_{\mathrm{i}}}^{\lambda_{\mathrm{f}}} E_{b \lambda} d \lambda$ is the energy flux radiated out by the blackbody at 
the same temperature in the atmospheric-window wavelength range. This is the maximum energy flux that an object at that temperature can emit. Thus, the first term in Eq. (1) is the ratio that quantitively describes the similarity of the designed structure to the ideal emissivity with respect to the energy flux. For the remaining wavelength range (i.e., $\lambda<$ $8 \mu \mathrm{m}$ and $13 \mu \mathrm{m}<\lambda$ ), the optimization strives to make the emissivity as low as possible. Therefore, in the definition of FOM, the coefficients are negative. Since the ideal FOM value is 1 and the worst FOM value is -2 , where the emissivity functions take exactly the opposite shape, our optimization task maximizes the FOM.

It should be noted that according to Kirchoff's law, the spectral emittance is equal to the spectral absorptance in the thermal equilibrium state. In the following calculations, the spectral emittance property is obtained directly from the spectral absorptance.

\section{Learning by FM}

Using a D-Wave quantum annealer, the lower energy states of QUBO can be effectively searched. QUBO with $N$ bits is given by

$$
\mathcal{H}=\sum_{i=1}^{N} \sum_{j=1}^{N} Q_{i j} q_{i} q_{j},
$$

where $q_{i}$ is the $0 / 1$ binary bit and $Q_{i j}=Q_{j i}$ takes a real value. The key to our idea is to use a machine learning regression model, which can be expressed by QUBO. Then the next candidate material structure with a high acquisition function can be rapidly selected by a quantum annealer. For the prediction, we utilize an FM, which is given by

$$
f(\mathbf{q})=\sum_{i=1}^{N} w_{i} q_{i}+\sum_{i=1}^{N} \sum_{j=1}^{N} \sum_{k=1}^{K} v_{i k} v_{j k} q_{i} q_{j},
$$

where $\mathbf{q}=\left\{q_{1}, \ldots, q_{N}\right\}$ determines the structure of the target metamaterial (Fig. 2), and $N=L \times(C+1)$. In this model, as the factorization size $K$ decreases, the number of fitting parameters $\left\{v_{i k}\right\}$ is reduced. Note that the $K=N$ case is equivalent to the regression model by QUBO itself. An FM with small $K$ should realize a good prediction without overfitting when the training dataset is small (see Supplemental note B in Ref. [56]). Thus, this model should be suitable for automated materials discovery due to the small number of training data.

In this paper, the factorization size is fixed as $K=8$, which is the default parameter in the LIBFM package [34,60,61]. In the training, a negative FOM is given as $f(\mathbf{q})$ to treat the maximization task of the FOM as the minimization by a quantum annealer. Some parameters $\left(\left\{w_{i}\right\}\right.$ and $\left.\left\{v_{i k}\right\}\right)$ are tuned to predict a negative FOM with a higher accuracy. That is, the predicted negative FOM is used as the acquisition function itself. In this paper, these parameters are determined by Adam (adaptive moment estimation) for the training dataset.

\section{Selection by the D-Wave quantum annealer}

A D-Wave 2000Q quantum annealer is utilized to select the next candidate material in the automated materi- als discovery. The D-Wave 2000Q has 2038 qubits on the chimera graph [62]. An FM is implemented on a fully connected graph, and the D-Wave 2000Q can create a graph with 63 nodes by regarding some qubits as one variable. The dwave.embedding.chimera.find_clique_embedding method [63] is used for this embedding. Although the maximum problem size for automated materials discovery using our algorithm is 63 , the fast selection from at most $2^{63}$ candidates is very attractive in materials science. The trained parameters $\left(\left\{w_{i}\right\}\right.$ and $\left.\left\{v_{i k}\right\}\right)$ in the FM are converted to the parameters $\left(\left\{Q_{i j}\right\}\right)$ in the QUBO format. If num_reads $=50$, which is the parameter for the D-Wave 2000Q [64], 50 states are outputted as the ground state candidates within $16 \mathrm{~ms}$ as the QPU time. Then the state with the lowest energy out of these 50 states is used as the next material structure candidate. After the candidate is selected, its FOM is evaluated by RCWA, and the number of training data for the FM increases. By repeating this procedure (Fig. 1), the metamaterial structure with a high FOM can be obtained with a small number of evaluations. So far, Ising machines, including quantum annealers, are experimentally utilized in the training processes for machine learning [28,29]. Consequently, our selection process is a new application of Ising machines.

Since our implementation does not exclude already observed structures as the candidates in the selection by a quantum annealer, it is possible to select an already observed structure. When such a situation occurs, a metamaterial structure is randomly selected from the unobserved structures as the next candidate.

\section{METAMATERIALS DESIGN BY FMQA}

\section{A. Performance of FMQA}

First, to clarify the usefulness of an FM as a regression model for our metamaterial design, the performances using an FM and a Gaussian process (GP) to find the best structure for the $L=4$ and $C=3$ case are compared. An exhaustive search of the acquisition function defined by the negative FOM can easily be performed on classical computers to select the next candidate material. Furthermore, a search where the next candidate material is randomly generated from all candidates in the selection part is also compared. In this case, since the number of candidate material structures is only $2^{16}=65536$, all the FOMs by RCWA are evaluated to identify the best structure.

Figure 3(a) shows the best FOM as a function of the number of calculated structures (iterations of the cycle depicted in Fig. 1) by each method. Here, 16 optimization runs with different initial choices are performed and the FOMs are averaged out. All three methods use the same first 50 randomly selected structures as the initial data. The regression results are used from step 51 for the FM and the GP cases. The results in order from worst to best are the random search, the GP, and the FM. Because the FM finds the best structure within 300 iterations, it is suitable for the regression model of our target. This result says that, at least, the learning by the FM is more useful than a random search to discover metamaterial structures with a high FOM within a small number of simulations. This result is promising for our metamaterial design. 
(a)

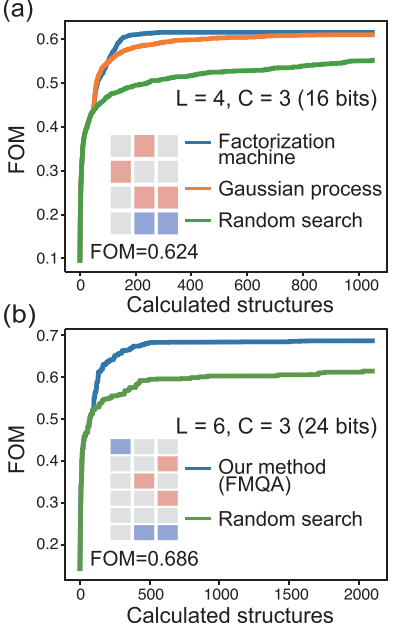

(c)

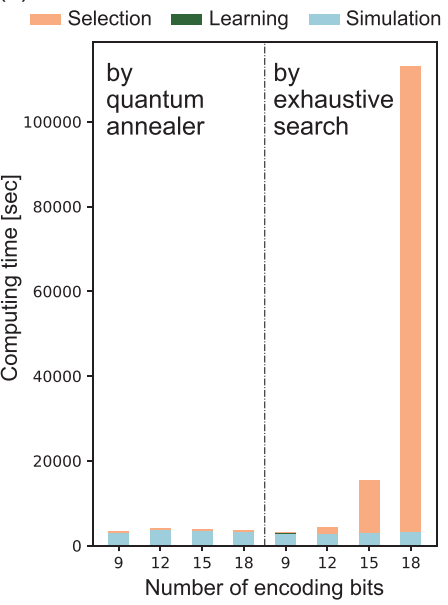

FIG. 3. (a) Dependence of the best FOM on the number of calculated structures (iterations) by automated materials discovery using an FM, a Gaussian process, and a random search for the $L=4$ and $C=3$ case. Inset is the optimum structure and its FOM. Blue, red, and gray squares denote $\mathrm{SiO}_{2}, \mathrm{SiC}$, and PMMA, respectively. (b) Best FOM by FMQA using a quantum annealer and the random search for the $L=6$ and $C=3$ case. Inset is the structure with the best FOM identified by FMQA. (c) Computing time to perform 500 iterations of automated materials discovery using a quantum annealer and an exhaustive search on a classical computer with an Intel Xeon E5-2690 v3 @ 2.6GHz for the selection part, respectively. Learnings and simulations are performed by the same classical computer.

Next, the problem with $L=6$ and $C=3$ by FMQA is considered using a quantum annealer. Since the candidate number is $2^{24}=16777216$, an evaluation of all FOMs predicted by regression models such as an FM and a GP to select the next candidate is a time-consuming task on classical computers. Thus, a more realistic option is to use a quantum annealer for the selection part rather than an exhaustive search. Figure 3(b) plots the best FOM by FMQA and a random search as functions of the iteration number. The average values from 16 independent runs with 50 different initial structures are plotted. FMQA can reduce the number of simulations to find a metamaterial structure with a higher FOM. Hence, it is a useful tool to design new metamaterials. Supplemental note C in Ref. [56] compares the effectiveness of a quantum annealer for FMQA to a local search method and simulated annealing.

Because the computing time to perform FMQA is important, the computing time of automated materials discovery based on an FM when the selection part is conducted by a quantum annealer is compared to an exhaustive search by a classical computer with an Intel Xeon E5-2690 v3 @ 2.6GHz. Note that to this point, the black-box optimization algorithms accompanied by acquisition functions (e.g., Bayesian optimization) have employed an exhaustive search for the selection part. Here, an exhaustive search is compared to the computing time between our algorithm and a conventional algorithm. Figure 3(c) plots the computing time to perform 500 iterations as a function of the problem size (number of encoding bits). The selection time, learning time by an FM, and simulation time by RCWA are separately illustrated. The target structure is the $L=3$ case with various $C=2,3,4$, or

5 , which are encoded using $9,12,15$, and 18 bits, respectively. Table S1 in Ref. [56] summarizes the empirical computing time.

Since the learning and simulation are conducted on the same classical computer, these parts require about the same time in both cases. The use of a quantum annealer reduces the selection time. Both methods provide the structure with the highest FOM within only 500 iterations, and the number of simulations can be reduced to find the best one. Note that if the FOMs of all structures are evaluated by the RCWA simulations for the 18 bits case to identify the best structure, the required time is more than ten times longer than the algorithm where the exhaustive search method is used for the selection part. Although RCWA is a relatively high-speed simulation method (one calculation takes one minute at most), the most time-consuming part becomes the simulation time when FMQA is performed. Consequently, a quantum annealer can be used to solve the hard computational barrier in the automated materials discovery.

\section{B. Optimum metamaterial structure search by FMQA}

We search the optimum structure of the metamaterial for radiative cooling. Varying the number of layers and columns of the target structure should help elucidate a trend to achieve a high FOM. Starting from the previous setting $(L=6$ and $C=3$ ), the number of layers $L$ is changed. The range of $L$ is varied from 3 to 9 , and a single run with the first 50 randomly generated initial structures and 2000 iterations is conducted for each optimization trial. Figure 4(a) plots the best FOMs as a function of the number of calculated structures for various numbers of layers. The structure with five layers $(L=5)$ exhibits the highest FOM. Figure 4(b) shows the found structure with the best FOM for each $L$. Interestingly, when $L \geqslant 6$, some layers only contain PMMA but removing the PMMA-only layers decreases the FOM. Consequently, the existence of the PMMA layer plays an important role in improving the FOM for thick metamaterials.

Next, the number of columns $C$ in the target structures is changed while the number of layers is fixed to five $(L=5)$. Figure 4(c) shows the best FOM as a function of the iteration number. Larger FOMs appear for the $C=4$ and 6 cases. Increasing the number of columns to more than seven $(C \geqslant 7)$ does not yield a higher FOM. Since larger cases are multiples of smaller ones with commensurate periods, a metamaterial structure for $C=8$ should have a similar FOM as the $C=4$ case. Thus 2000 samplings are too small to find the optimum structure with a higher FOM due to the massive number of candidates for $C=8$. In fact, the change in the FOMs with the number of calculated structures gradually increases for larger systems [Fig. 4(c)]. Consequently, searches should be continued to find the best structure for larger systems.

Figure 4(b) summarizes the metamaterial structures with a high FOM found by FMQA for various $L$ and $C$ values. The structure for the $L=5$ and $C=6$ case has the highest FOM, and its value is 0.724 for radiative cooling. Figure 4(d) shows the emissive power of the structure. The large emittances fall into the atmospheric window. Note that the maximum FOM of a multilayer structure constructed by $\mathrm{SiO}_{2}, \mathrm{SiC}$, and PMMA is 0.250 for five layers. In the multilayer structure, 
(a)
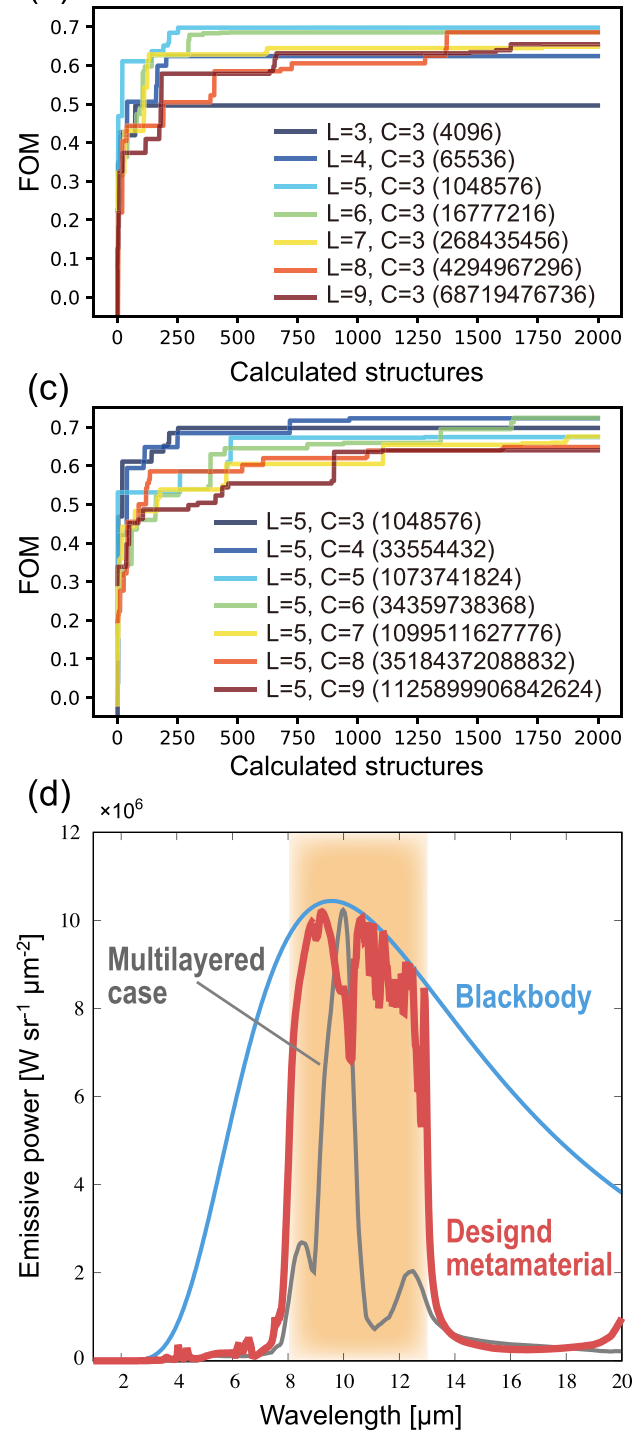

(b)

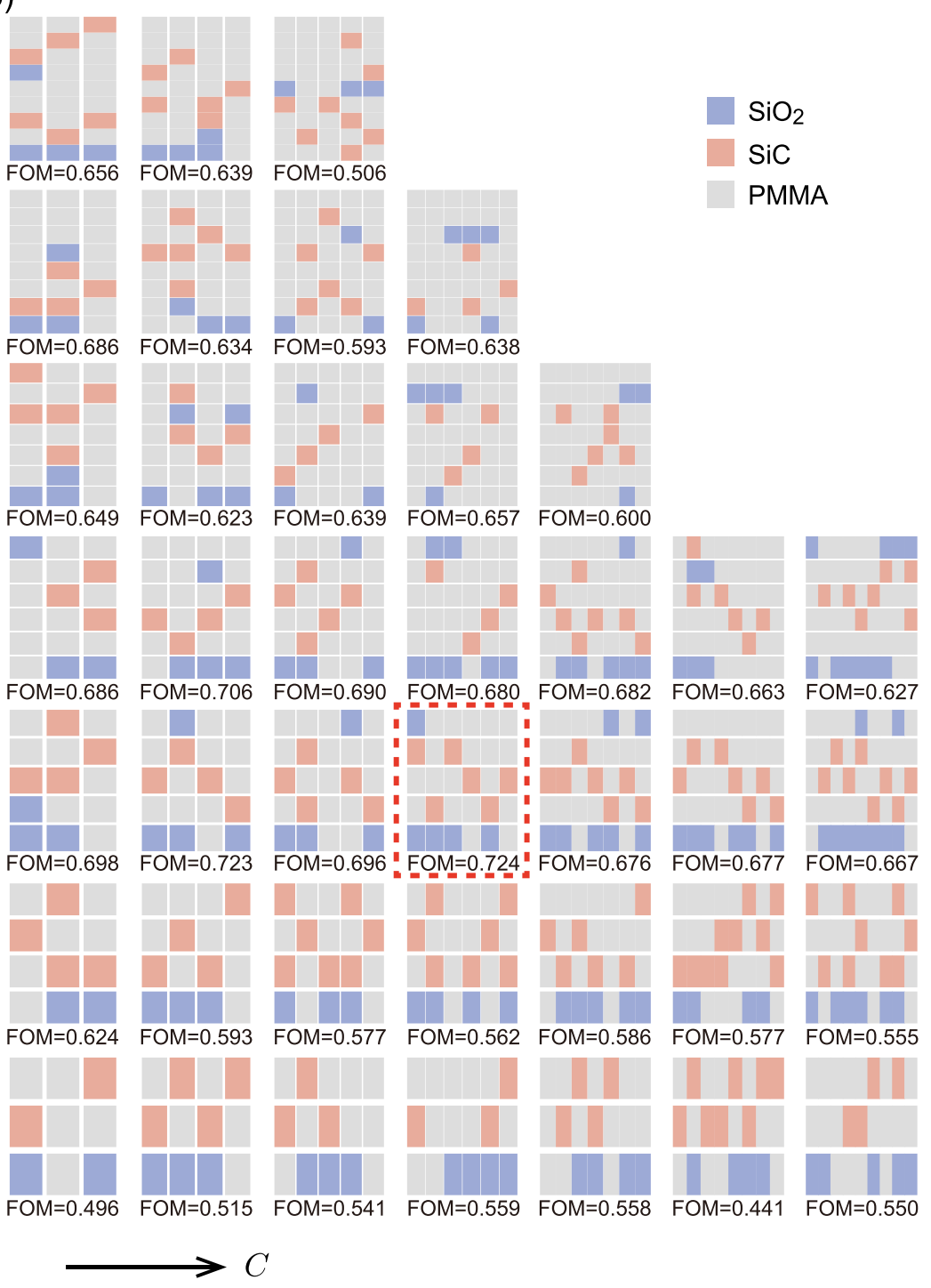

FIG. 4. (a) Best FOM as a function of the iteration number for $C=3$ with various $L$. Plotted results are for a single run with the first 50 randomly generated initial structures. Parentheses denote the number of candidates. (b) Structures with a high FOM designed by FMQA depending on $L$ and $C$. Red dotted line denotes the structure with the highest FOM in our search. (c) Change in the best FOM for $L=5$ with various $C$ obtained by a single run with the first 50 randomly generated initial structures. (d) Emissive power calculated by RCWA (red curve) of the designed structure with the highest FOM for the $L=5$ and $C=6$ case, which is surrounded by the red dotted line in (b). For comparison, the blackbody emissive power (blue curve) and the multilayer optimum case with five layers (grey curve) are shown (see Supplemental note D in Ref. [56]).

each planar layer is constructed by the same material similar to the $C=1$ case. Hence, the material arrangement in the $z$ direction is optimized. (For the detail optimized structure, see Supplemental note D in Ref. [56].) This means that our designed metamaterial structure is essential to obtain a high FOM for radiative cooling.

\section{Mechanism of high emittance in designed metamaterial}

From the list of optimized structures [Fig. 4(b)], structures with $\mathrm{SiO}_{2}$ located separately at the top and bottom where $\mathrm{SiC}$ mediates the middle part always show a higher FOM. To understand which part of the structure absorbs the wave energy, the electric power dissipation density $w_{e}$ of each part is evaluated. This value is calculated as [65]

$$
w_{e}=\frac{1}{2} \epsilon_{0} \epsilon_{\operatorname{Im}} \omega|\mathbf{E}|^{2}
$$

where $\epsilon_{0}$ is the permittivity in a vacuum, $\epsilon_{\operatorname{Im}}$ is the imaginary part of the dielectric function, $\omega$ is the angular frequency, and $\mathbf{E}$ is the complex electric field calculated by RCWA.

Figure 5(a) shows $w_{e}$ for several typical wavelengths of the optimum structure designed above [i.e., the metamaterial structure surrounded by the red dotted line in Fig. 4(b)]. The top and bottom $\mathrm{SiO}_{2}$ absorb most of the wave energy within 8-11 $\mu \mathrm{m}$, whereas the middle parts of the SiC layers dominate the absorption between 11-13 $\mu \mathrm{m}$. Furthermore, around $11.8 \mu \mathrm{m}, \mathrm{SiO}_{2}$ also facilitates absorption. 
(a) Normalized electric power dissipation density
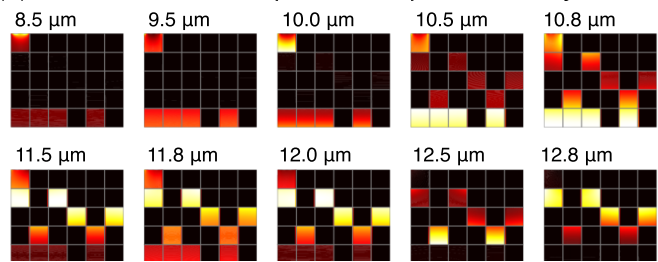

$11.8 \mu \mathrm{m}$
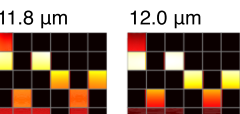

$12.5 \mu \mathrm{m}$
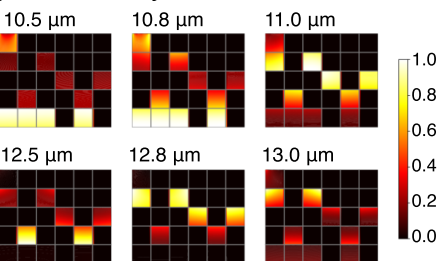

(b) Normalized magnetic field
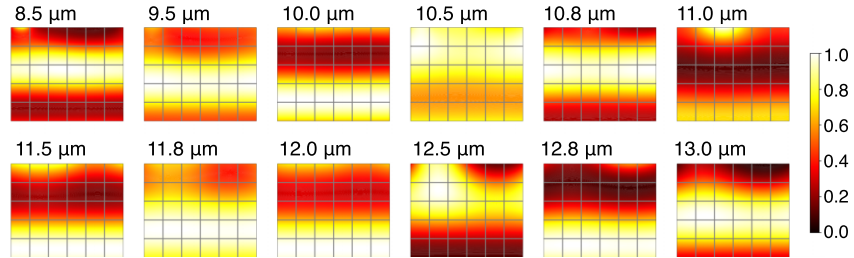

FIG. 5. Contour plots of (a) the normalized electric power dissipation density at select wavelengths and (b) the normalized magnetic field for the designed optimum structure with $L=5$ and $C=6$ which is shown in Fig. 4(b).

Next, the mechanism of the high emittance of the designed metamaterial is examined. The emittance contour plot of the $p$-wave dispersion relation indicates that a high emittance is almost insensitive against the incident angle (see Supplemental note $\mathrm{E}$ in Ref. [56]). Hence, the resonance is not due to the surface phonon polariton, which is highly sensitive to the incident angle. On the other hand, in terms of the magnetic polariton [66], the diamagnetic response between the external field and centralized magnetic field inside the structure is usually excited. Consequently, the magnetic polariton is almost insensitive of the incident angle.

To further elucidate the magnetic polariton resonance, Fig. 5(b) plots the magnetic field normalized by the maximum value at several typical wavelengths. Comparing Figs. 4(d) and 5(b) reveals that the magnetic fields always show a strong confinement at the part with a high emittance, which is where the polariton resonance is excited. Furthermore, when the emittance curve falls, the confined magnetic field becomes flatter and less centralized. These results suggest that the high emittance of the designed structure originates from the magnetic polariton resonance. Since a multilayer structure with five layers only shows a narrow absorption band between 8.5 and $10.5 \mu \mathrm{m}$ [Fig. 4(d)], the structure design of materials is critical to confine the magnetic field. To promote the understanding of our designed thermal radiator, the angle dependence and theoretical cooling power analysis are discussed in Supplemental notes E and F in Ref. [56].

\section{DISCUSSION AND SUMMARY}

In summary, we have proposed a new optimization technique called FMQA, which uses a quantum annealer for automated materials discovery. In our algorithm, the next candidate material, which is selected with respect to the acquisition function, is represented as a solution to a combinatorial optimization by using an FM, and this optimization problem is solved by a quantum annealer. By performing FMQA using the D-Wave 2000Q quantum annealer, we have demonstrated that a metamaterial can be designed for radiative cooling within a small number of RCWA simulations. In the target metamaterial, which has $\mathrm{SiO}_{2}$ and $\mathrm{SiC}$ wires placed in PMMA, a high FOM of 0.724 is achieved for radiative sky cooling. Compared to previously designed structures, the targeted single polarization FOM is far greater than the best reported structures with a comparable polarization- and angle-averaged cooling power (see Supplemental note F in Ref. [56]).

Although fabrication is beyond the scope of this paper, a stratified structure similar to the designed structures has been prepared using current technology [67]. Hence, it should be possible to fabricate the designed optimal structure. The results will be discussed elsewhere. Here, we demonstrate that our method is applicable to the case where the metamaterial design is expressed by binary bits. However, if combined with methods for encoding integer variables $[68,69]$, our algorithm is applicable to discrete structural and compositional optimizations of any property as long as the property calculation is relatively fast with respect to the optimization process. Therefore our algorithm should help manage the transportation of various carriers (e.g., phonons, electrons, and magnons) and contribute to creation of new energy materials by combining with a straight-forward extension such as tailoring spectral and angular-dependent radiative heat transfer.

Ising machines are conventionally used to optimize explicitly defined functions. Herein quantum annealer as an Ising machine is used for the black-box optimization algorithm. In the future, the application domain of our algorithm will expand to even larger problems as next-generation quantum annealers or other Ising machines equipped with many bits [70-76] become available. As demonstrated by our experiments, the hard computational barrier (e.g., candidate selection) in automated materials discovery can be partly resolved with the help of an Ising machine. Accordingly, the most time-consuming part will be the simulation of material properties even if a fast simulation on classical computers is used. Therefore it is essential to speed up the materials simulations. As there are increasing number of studies on materials simulations by quantum computing to overcome this problem [77-86], we expect in the future that our algorithm will further accelerate materials discovery.

\section{ACKNOWLEDGMENTS}

We thank Kei Terayama, Masato Sumita, and Kotaro Tanahashi for the useful discussions. This article is based on the results obtained from a project subsidized by the "Materials Research by Information Integration" Initiative (MI2I) project and Core Research for Evolutional Science and Technology (CREST) (Grants No. JPMJCR1502, No. JPMJCR16Q5, and No. JPMJCR17J2) from the Japan Science and Technology Agency (JST). This work is also supported partially by KAKENHI Grants (Grants No. 15K17720, No. 15H03699, No. 16H04274, and No. 19H01553) from the Japan Society for the Promotion of Science (JSPS). S.T. is supported by JST PRESTO (Grant No. JPMJPR1665). K.T. is supported by NEDO P15009, SIP (Technologies for Smart Bio-industry and Agriculture) and JST PRISM JPMJCR18Y3. 
[1] M. A. Green, Commercial progress and challenges for photovoltaics, Nat. Energy 1, 15015 (2016).

[2] A. P. Raman, M. A. Anoma, L. Zhu, E. Rephaeli, and S. Fan, Passive radiative cooling below ambient air temperature under direct sunlight, Nature (London) 515, 540 (2014).

[3] A. Sakurai, K. Yada, T. Simomura, S. Ju, M. Kashiwagi, H. Okada, T. Nagao, K. Tsuda, and J. Shiomi, Ultranarrowband wavelength-selective thermal emission with aperiodic multilayered metamaterials designed by bayesian optimization, ACS Cent. Sci. 5, 391 (2019).

[4] Q. Pang, X. Liang, C. Y. Kwok, and L. F. Nazar, Advances in lithium-sulfur batteries based on multifunctional cathodes and electrolytes, Nat. Energy 1, 16132 (2016).

[5] A. Majumdar, Thermoelectricity in semiconductor nanostructures, Science 303, 777 (2004).

[6] J. C. Knight, Photonic crystal fibres, Nature (London) 424, 847 (2003).

[7] J. Ravichandran, A. K. Yadav, R. Cheaito, P. B. Rossen, A. Soukiassian, S. J. Suresha, J. C. Duda, B. M. Foley, C.-H. Lee, and Y. Zhu, Crossover from incoherent to coherent phonon scattering in epitaxial oxide superlattices, Nat. Mater. 13, 168 (2014).

[8] E. Bocquillon, V. Freulon, J.-M. Berroir, P. Degiovanni, B. Plaçais, A. Cavanna, Y. Jin, and G. Fève, Coherence and indistinguishability of single electrons emitted by independent sources, Science 339, 1054 (2013).

[9] J. Xiao, G. E. W. Bauer, K.-C. Uchida, E. Saitoh, and S. Maekawa, Theory of magnon-driven spin Seebeck effect, Phys. Rev. B 81, 214418 (2010).

[10] S. Ju, T. Shiga, L. Feng, Z. Hou, K. Tsuda, and J. Shiomi, Designing Nanostructures for Phonon Transport Via Bayesian Optimization, Phys. Rev. X 7, 021024 (2017).

[11] K. T. Butler, D. W. Davies, H. Cartwright, O. Isayev, and A. Walsh, Machine learning for molecular and materials science, Nature (London) 559, 547 (2018).

[12] M. Sumita, X. Yang, S. Ishihara, R. Tamura, and K. Tsuda, Hunting for organic molecules with artificial intelligence: molecules optimized for desired excitation energies, ACS Cent. Sci. 4, 1126 (2018).

[13] T. M. Dieb, S. Ju, K. Yoshizoe, Z. Hou, J. Shiomi, and K. Tsuda, MDTS: Automatic complex materials design using Monte Carlo tree search, Sci. Tech. Adv. Mater. 18, 498 (2017).

[14] W. Paszkowicz, Genetic algorithms, a nature-inspired tool: Survey of applications in materials science and related fields, Mater. Manuf. Process. 24, 174 (2009).

[15] M. W. Johnson, M. H. Amin, S. Gildert, T. Lanting, F. Hamze, N. Dickson, R. Harris, A. J. Berkley, J. Johansson, and P. Bunyk, Quantum annealing with manufactured spins, Nature (London) 473, 194 (2011).

[16] T. Kadowaki and H. Nishimori, Quantum annealing in the transverse Ising model, Phys. Rev. E 58, 5355 (1998).

[17] S. Tanaka, R. Tamura, and B. K. Chakrabarti, Quantum Spin Glasses, Annealing and Computation (Cambridge University Press, Cambridge, 2017).

[18] T. Albash and D. A. Lidar, Adiabatic quantum computation, Rev. Mod. Phys. 90, 015002 (2018).

[19] S. Boixo, T. Albash, F. M. Spedalieri, N. Chancellor, and D. A. Lidar, Experimental signature of programmable quantum annealing, Nat. Commun. 4, 2067 (2013).
[20] S. Boixo, T. F. Rønnow, S. V. Isakov, Z. Wang, D. Wecker, D. A. Lidar, J. M. Martinis, and M. Troyer, Evidence for quantum annealing with more than one hundred qubits, Nat. Phys. 10, 218 (2014).

[21] T. F. Rønnow, Z. Wang, J. Job, S. Boixo, S. V. Isakov, D. Wecker, J. M. Martinis, D. A. Lidar, and M. Troyer, Defining and detecting quantum speedup, Science 345, 420 (2014).

[22] T. Albash, W. Vinci, A. Mishra, P. A. Warburton, and D. A. Lidar, Consistency tests of classical and quantum models for a quantum annealer, Phys. Rev. A 91, 042314 (2015).

[23] V. S. Denchev, S. Boixo, S. V. Isakov, N. Ding, R. Babbush, V. Smelyanskiy, J. Martinis, and H. Neven, What is the Computational Value of Finite-Range Tunneling? Phys. Rev. X 6, 031015 (2016).

[24] S. V. Isakov, G. Mazzola, V. N. Smelyanskiy, Z. Jiang, S. Boixo, H. Neven, and M. Troyer, Understanding Quantum Tunneling through Quantum Monte Carlo Simulations, Phys. Rev. Lett. 117, 180402 (2016).

[25] S. Mandrà, Z. Zhu, W. Wang, A. Perdomo-Ortiz, and H. G. Katzgraber, Strengths and weaknesses of weak-strong cluster problems: A detailed overview of state-of-the-art classical heuristics versus quantum approaches, Phys. Rev. A 94, 022337 (2016).

[26] A. Perdomo-Ortiz, N. Dickson, M. Drew-Brook, G. Rose, and A. Aspuru-Guzik, Finding low-energy conformations of lattice protein models by quantum annealing, Sci. Rep. 2, 571 (2012).

[27] R. Y. Li, R. Di Felice, R. Rohs, and D. A. Lidar, Quantum annealing versus classical machine learning applied to a simplified computational biology problem, npj Quantum Inf. 4, 14 (2018).

[28] S. H. Adachi and M. P. Henderson, Application of quantum annealing to training of deep neural networks, arXiv:1510.06356.

[29] M. Benedetti, J. Realpe-Gómez, R. Biswas, and A. PerdomoOrtiz, Estimation of effective temperatures in quantum annealers for sampling applications: A case study with possible applications in deep learning, Phys. Rev. A 94, 022308 (2016).

[30] D. O’Malley, V. V. Vesselinov, B. S. Alexandrov, and L. B. Alexandrov, Nonnegative/binary matrix factorization with a DWave quantum annealer, PLoS ONE 13, e0206653 (2018).

[31] F. Neukart, G. Compostella, C. Seidel, D. Von Dollen, S. Yarkoni, and B. Parney, Traffic flow optimization using a quantum annealer, Front. ICT 4, 29 (2017).

[32] E. Boyda, S. Basu, S. Ganguly, A. Michaelis, S. Mukhopadhyay, and R. R. Nemani, Deploying a quantum annealing processor to detect tree cover in aerial imagery of California, PLoS ONE 12, e0172505 (2017).

[33] M. Ohzeki, A. Miki, M. J. Miyama, and M. Terabe, Control of automated guided vehicles without collision by quantum annealer and digital devices, Front. Comput. Sci. 1, 9 (2019).

[34] S. Rendle, Factorization machines, in Proceedings of the 2010 IEEE International Conference on Data Mining (IEEE, Piscataway, 2010), pp. 995-1000.

[35] Our implementation (FMQA) is available on GitHub at https://github.com/tsudalab/fmqa/.

[36] M. De Zoysa, T. Asano, K. Mochizuki, A. Oskooi, T. Inoue, and S. Noda, Conversion of broadband to narrowband thermal emission through energy recycling, Nat. Photon. 6, 535 (2012).

[37] D. M. Bierman, A. Lenert, W. R. Chan, B. Bhatia, I. Celanović, M. Soljačić, and E. N. Wang, Enhanced photovoltaic energy conversion using thermally based spectral shaping, Nat. Energy 1, 16068 (2016). 
[38] O. Ilic, P. Bermel, G. Chen, J. D. Joannopoulos, I. Celanovic, and M. Soljačić, Tailoring high-temperature radiation and the resurrection of the incandescent source, Nat. Nanotechnol. 11, 320 (2016).

[39] C. Wu, A. B. Khanikaev, R. Adato, N. Arju, A. A. Yanik, H. Altug, and G. Shvets, Fano-resonant asymmetric metamaterials for ultrasensitive spectroscopy and identification of molecular monolayers, Nat. Mater. 11, 69 (2012).

[40] S. Luo, J. Zhao, D. Zuo, and X. Wang, Perfect narrow band absorber for sensing applications, Opt. Express 24, 9288 (2016).

[41] X. L. Liu, L. P. Wang, and Z. M. Zhang, Wideband tunable omnidirectional infrared absorbers based on doped-silicon nanowire arrays, J. Heat Transf. 135, 061602 (2013).

[42] K. Du, Q. Li, W. Zhang, Y. Yang, and M. Qiu, Wavelength and thermal distribution selectable microbolometers based on metamaterial absorbers, IEEE Photon. J. 7, 6800908 (2015).

[43] N. I. Landy, C. M. Bingham, T. Tyler, N. Jokerst, D. R. Smith, and W. J. Padilla, Design, theory, and measurement of a polarization-insensitive absorber for terahertz imaging, Phys. Rev. B 79, 125104 (2009).

[44] J. Obando, Y. Cadavid, and A. Amell, Theoretical, experimental and numerical study of infrared radiation heat transfer in a drying furnace, Appl. Therm. Eng. 90, 395 (2015).

[45] A. R. Gentle and G. B. Smith, Radiative heat pumping from the earth using surface phonon resonant nanoparticles, Nano Lett. 10, 373 (2010).

[46] A. R. Gentle and G. B. Smith, A subambient open roof surface under the Mid-Summer sun, Adv. Sci. 2, 1500119 (2015).

[47] M. M. Hossain, B. Jia, and M. Gu, A metamaterial emitter for highly efficient radiative cooling, Adv. Opt. Mater. 3, 1047 (2015).

[48] Z. Chen, L. Zhu, A. Raman, and S. Fan, Radiative cooling to deep sub-freezing temperatures through a 24-h day-night cycle, Nat. Commun. 7, 13729 (2016)

[49] X. Sun, Y. Sun, Z. Zhou, M. A. Alam, and P. Bermel, Radiative sky cooling: Fundamental physics, materials, structures, and applications, Nanophotonics 6, 997 (2017).

[50] C. Zou, G. Ren, M. M. Hossain, S. Nirantar, W. Withayachumnankul, T. Ahmed, M. Bhaskaran, S. Sriram, M. Gu, and C. Fumeaux, Metal-loaded dielectric resonator metasurfaces for radiative cooling, Adv. Opt. Mater. 5, 1700460 (2017).

[51] K. Sun, C. A. Riedel, Y. Wang, A. Urbani, M. Simeoni, S. Mengali, M. Zalkovskij, B. Bilenberg, C. H. De Groot, and O. L. Muskens, Metasurface optical solar reflectors using AZO transparent conducting oxides for radiative cooling of spacecraft, ACS Photon. 5, 495 (2017).

[52] Y. Zhai, Y. Ma, S. N. David, D. Zhao, R. Lou, G. Tan, R. Yang, and X. Yin, Scalable-manufactured randomized glass-polymer hybrid metamaterial for daytime radiative cooling, Science $\mathbf{3 5 5}$, 1062 (2017).

[53] M. Zeyghami, D. Y. Goswami, and E. Stefanakos, A review of clear sky radiative cooling developments and applications in renewable power systems and passive building cooling, Sol. Energy Mater. Sol. Cells 178, 115 (2018).

[54] A. Hervé, J. Drévillon, Y. Ezzahri, and K. Joulain, Radiative cooling by tailoring surfaces with microstructures: Association of a grating and a multi-layer structure, J. Quant. Spectrosc. Radiat. Transfer 221, 155 (2018).
[55] S. Atiganyanun, J. B. Plumley, S. J. Han, K. Hsu, J. Cytrynbaum, T. L. Peng, S. M. Han, and S. E. Han, Effective radiative cooling by paint-format microsphere-based photonic random media, ACS Photon. 5, 1181 (2018).

[56] See Supplemental Material at http://link.aps.org/supplemental/ 10.1103/PhysRevResearch.2.013319 for Supplemental Table I. Empirical computing time in our automated materials discovery; Supplemental note A. Validation of RCWA calculation; Supplemental note B. Dependence of an FM on the factorization size; Supplemental note C. Comparison between a quantum annealer, simulated annealing, and local search; Supplemental note D. FOM in multilayer materials; Supplemental note E. Angle dependence of the designed thermal radiator; and Supplemental note F. Performance comparison with previous works.

[57] M. N. Polyanskiy, Refractive index database, https://refractiveindex.info.

[58] E. D. Palik, Handbook of Optical Constants of Solids (Academic Press, Burlington, 1997).

[59] K. Han and C.-H. Chang, Numerical modeling of subwavelength anti-reflective structures for solar module applications, Nanomaterials 4, 87 (2014).

[60] S. Rendle, Factorization machines with libfm, ACM Trans. Intell. Syst. Technol. 3, 57 (2012).

[61] http://www.libfm.org/ (accessed 24th October 2018).

[62] https://www.dwavesys.com/d-wave-two-system (accessed 1 January 2019).

[63] https://docs.ocean.dwavesys.com/projects/system/en/stable/ reference/generated/dwave.embedding.chimera.find_clique_ embedding.html (accessed 1st October 2018).

[64] https://docs.dwavesys.com/docs/latest/c_solver_1.html (accessed 1 January 2019).

[65] J. M. Zhao and Z. M. Zhang, Electromagnetic energy storage and power dissipation in nanostructures, J. Quant. Spectrosc. Radiat. Transfer 151, 49 (2015).

[66] L. P. Wang and Z. M. Zhang, Phonon-mediated magnetic polaritons in the infrared region, Opt. Express 19, A126 (2011).

[67] D. M. Chambers, G. P. Nordin, and S. Kim, Fabrication and analysis of a three-layer stratified volume diffractive optical element high-efficiency grating, Opt. Express 11, 27 (2003).

[68] S. Hadfield, Z. Wang, B. O’Gorman, E. G. Rieffel, D. Venturelli, and R. Biswas, From the quantum approximate optimization algorithm to a quantum alternating operator ansatz, Algorithms 12, 34 (2019).

[69] N. Chancellor, Domain wall encoding of discrete variables for quantum annealing and QAOA, Quantum Sci. Technol. 4, 045004 (2019).

[70] M. Yamaoka, C. Yoshimura, M. Hayashi, T. Okuyama, H. Aoki, and H. Mizuno, A 20k-spin Ising chip to solve combinatorial optimization problems with CMOS annealing, IEEE J. SolidState Circuits 51, 303 (2016).

[71] G. Rosenberg, P. Haghnegahdar, P. Goddard, P. Carr, K. Wu, and M. L. De Prado, Solving the optimal trading trajectory problem using a quantum annealer, IEEE J. Sel. Top. Signal Proc. 10, 1053 (2016).

[72] T. Inagaki, Y. Haribara, K. Igarashi, T. Sonobe, S. Tamate, T. Honjo, A. Marandi, P. L. McMahon, T. Umeki, and K. Enbutsu, A coherent Ising machine for 2000-node optimization problems, Science 354, 603 (2016). 
[73] P. L. McMahon, A. Marandi, Y. Haribara, R. Hamerly, C. Langrock, S. Tamate, T. Inagaki, H. Takesue, S. Utsunomiya, and K. Aihara, A fully programmable 100-spin coherent Ising machine with all-to-all connections, Science 354, 614 (2016).

[74] S. Matsubara, H. Tamura, M. Takatsu, D. Yoo, B. Vatankhahghadim, H. Yamasaki, T. Miyazawa, S. Tsukamoto, Y. Watanabe, and K. Takemoto, Ising-model optimizer with parallel-trial bit-sieve engine, in Conference on Complex, Intelligent, and Software Intensive Systems, edited by L. Barolli and O. Terzo (Springer, Cham, 2017), pp. 432-438.

[75] H. Goto, K. Tatsumura, and A. R. Dixon, Combinatorial optimization by simulating adiabatic bifurcations in nonlinear Hamiltonian systems, Sci. Adv. 5, eaav2372 (2019).

[76] T. Okuyama, T. Sonobe, K.-I. Kawarabayashi, and M. Yamaoka, Binary optimization by momentum annealing, Phys. Rev. E 100, 012111 (2019).

[77] A. Aspuru-Guzik, A. D. Dutoi, P. J. Love, and M. Head-Gordon, Simulated quantum computation of molecular energies, Science 309, 1704 (2005).

[78] B. P. Lanyon, J. D. Whitfield, G. G. Gillett, M. E. Goggin, M. P. Almeida, I. Kassal, J. D. Biamonte, M. Mohseni, B. J. Powell, and M. Barbieri, Towards quantum chemistry on a quantum computer, Nat. Chem. 2, 106 (2010).

[79] I. M. Georgescu, S. Ashhab, and F. Nori, Quantum simulation, Rev. Mod. Phys. 86, 153 (2014).

[80] A. Peruzzo, J. McClean, P. Shadbolt, M.-H. Yung, X.-Q. Zhou, P. J. Love, A. Aspuru-Guzik, and J. L. O'brien, A variational eigenvalue solver on a photonic quantum processor, Nat. Commun. 5, 4213 (2014).
[81] K. Sugisaki, S. Yamamoto, S. Nakazawa, K. Toyota, K. Sato, D. Shiomi, and T. Takui, Quantum chemistry on quantum computers: A polynomial-time quantum algorithm for constructing the wave functions of open-shell molecules, J. Phys. Chem. A 120, 6459 (2016).

[82] P. J. J. O’Malley, R. Babbush, I. D. Kivlichan, J. Romero, J. R. McClean, R. Barends, J. Kelly, P. Roushan, A. Tranter, N. Ding, B. Campbell, Y. Chen, Z. Chen, B. Chiaro, A. Dunsworth, A. G. Fowler, E. Jeffrey, E. Lucero, A. Megrant, J. Y. Mutus, M. Neeley, C. Neill, C. Quintana, D. Sank, A. Vainsencher, J. Wenner, T. C. White, P. V. Coveney, P. J. Love, H. Neven, A. Aspuru-Guzik, and J. M. Martinis, Scalable Quantum Simulation of Molecular Energies, Phys. Rev. X 6, 031007 (2016).

[83] A. Kandala, A. Mezzacapo, K. Temme, M. Takita, M. Brink, J. M. Chow, and J. M. Gambetta, Hardware-efficient variational quantum eigensolver for small molecules and quantum magnets, Nature (London) 549, 242 (2017).

[84] C. Hempel, C. Maier, J. Romero, J. McClean, T. Monz, H. Shen, P. Jurcevic, B. P. Lanyon, P. Love, R. Babbush, A. Aspuru-Guzik, R. Blatt, and C. F. Roos, Quantum Chemistry Calculations On a Trapped-ion Quantum Simulator, Phys. Rev. X 8, 031022 (2018).

[85] A. D. King, J. Carrasquilla, J. Raymond, I. Ozfidan, E. Andriyash, A. Berkley, M. Reis, T. Lanting, R. Harris, and F. Altomare, Observation of topological phenomena in a programmable lattice of 1,800 qubits, Nature (London) 560, 456 (2018).

[86] K. Mitarai, T. Yan, and K. Fujii, Generalization of the Output of a Variational Quantum Eigensolver by Parameter Interpolation with a Low-depth Ansatz, Phys. Rev. Appl. 11, 044087 (2019). 